\title{
Schäfer, Armin, und Michael Zürn (2021): Die demokratische Regression. Die politischen Ursachen des autoritären Populismus
}

\author{
Berlin: Suhrkamp. 247 Seiten. 16,00€
}

\section{Sören Hilbrich}

Angenommen: 29. Juni 2021 / Online publiziert: 19. Juli 2021

(C) Deutsche Vereinigung für Politikwissenschaft 2021

„Die demokratische Regression“ bringt zentrale Themen der langjährigen wissenschaftlichen Tätigkeit der beiden Autoren - Armin Schäfers Forschung zur abnehmenden Responsivität der Parlamente und Michael Zürns Forschung zu den Demokratiedefiziten politischer Institutionen jenseits der Nationalstaaten - zusammen und argumentiert dafür, dass beides zusammen den in den letzten Jahren zu beobachtenden Aufstieg autoritär-populistischer Parteien und Politiker*innen erklären kann. Damit wollen Schäfer und Zürn die verbreiteten sozioökonomischen und soziokulturellen Erklärungen für das Erstarken populistischer Parteien um eine genuin politische Erklärung ergänzen, die auf Schwächen in der Funktionsweise der Demokratien verweist.

Schäfer und Zürn zeigen zunächst unter Bezugnahme auf verschiedene Indikatoren der empirischen Demokratiemessung, dass man tatsächlich von einer demokratischen Regression sprechen kann. Sowohl die Qualität der Demokratie in vielen Ländern als auch die Anzahl der Länder, die überhaupt als Demokratien bezeichnet werden sollten, nahm in den letzten Jahren ab. Diese Entwicklung steht nach Auffassung der Autoren mit dem Entstehen einer neuen politischen Konfliktlinie in Verbindung, die seit den 1980er-Jahren zunehmend neben die traditionelle LinksRechts-Achse des Gegensatzes zwischen Arbeit und Kapital trat. Nach Schäfer und Zürn verläuft diese neue Konfliktlinie zwischen einem von den ,liberalen Globalisten“ (S. 80) vertretenen Kosmopolitismus und einer kommunitaristischen Gegenposition, die zurzeit in der öffentlichen politischen Auseinandersetzung hauptsächlich von autoritär-populistischen Parteien eingenommen wird.

Für das Erstarken populistischer Parteien machen Schäfer und Zürn Defizite der bestehenden politischen Systeme der Demokratien verantwortlich. Erstens nahm

Sören Hilbrich $(\bowtie)$

Deutsches Institut für Entwicklungspolitik (DIE), Bonn, Deutschland

E-Mail: Soeren.Hilbrich@die-gdi.de 
die Responsivität politischer Entscheidungen gegenüber den politischen Einstellungen der Bürger*innen mit geringer Bildung oder wenig Einkommen in den letzten Jahrzehnten aufgrund der Zusammensetzung der Parlamente ab. Zweitens wurden Kompetenzen von den Parlamenten auf sogenannte nichtmajoritäre Institutionen wie Zentralbanken, Verfassungsgerichten oder internationale Institutionen übertragen. Die Entscheidungsträger*innen dieser Institutionen werden jedoch nicht von den Bürger*innen gewählt und sind nicht gegenüber ihnen rechenschaftspflichtig. Das Gefühl vieler Unterstützer*innen populistischer Parteien, im politischen System kein Gehör zu finden, ist nach Schäfer und Zürn also nicht unbegründet.

Obwohl die Autoren den Aufstieg autoritär-populistischer Parteien auf tatsächliche Defizite der Demokratien zurückführen, verbessern populistische Parteien, sowohl wenn sie an die Macht gelangen als auch wenn sie in der Opposition sind, keineswegs die Qualität der Demokratie. Im Gegenteil, populistische Parteien beschädigen die Demokratie weiter, sie ,,sind selbst eine Triebkraft der demokratischen Regression“ (S. 127). Die Krisen der vergangenen Jahre - von der Finanzkrise über die „Integrationskrise“ (S. 151) infolge der gestiegenen Zahl von Geflüchteten in Europa bis zur Covid-19-Pandemie - wirkten als „Lupe und Brennglas“ (S. 153-154), die die Schwächen des politischen Systems zugleich sichtbar machen und ihre Folgen verstärken. Dazu, wie der demokratischen Regression entgegengewirkt werden kann, machen Schäfer und Zürn zehn (mehr oder weniger) konkrete Vorschläge. Beispielsweise schlagen sie vor, die Rekrutierungsmuster der Parteien zu verändern, nichtmajoritäre Institutionen stärker demokratisch zu kontrollieren und für kosmopolitische Positionen offener einzutreten, ohne die Verantwortung für unpopuläre Maßnahmen auf internationale Institutionen abzuschieben.

Mit ihrer empirisch fundierten Analyse fügen Schäfer und Zürn der Forschung zur hochrelevanten Frage nach den Ursachen des Aufstiegs autoritär-populistischer Parteien eine zweifellos interessante und wichtige Perspektive hinzu. Dennoch bleiben (natürlich) offene Fragen. Eine dieser Fragen bezieht sich auf die Reichweite ihrer Argumentation. In ihren Beispielen nennen Schäfer und Zürn auch populistische Parteien und Politiker*innen in Ländern wie Brasilien, Indien, der Türkei oder Venezuela. Doch dass die beschriebenen Entwicklungen in diesen Ländern genauso stattgefunden haben wie in Europa, ist nicht selbstverständlich. So erwähnen die Autoren selber, dass nichtmajoritäre Institutionen oberhalb der Ebene der Nationalstaaten (anders als nationale nichtmajoritäre Institutionen) in einem besonders großen Ausmaß innerhalb der EU-Kompetenzen auf Kosten der Parlamente hinzugewannen. Die Frage, ob die Responsivität der Parlamente gegenüber den Einstellungen von weniger gebildeten und einkommensschwachen Bürger*innen sich außerhalb von Europa und den USA im fraglichen Zeitraum ebenfalls verschlechtert hat, bleibt bei Schäfer und Zürn - wohl aus Mangel an Daten - offen.

Zudem stellt sich die Frage, warum gerade die in den letzten Jahren erfolgreichen autoritär-populistischen Parteien am besten in der Lage waren, aus den Schwächen der Funktionsweise der Demokratien Kapital zu schlagen. Schäfer und Zürn argumentieren dafür, dass wenig gebildete oder einkommensschwache Bürger*innen das (berechtigte) Gefühl haben, im politischen System nicht repräsentiert zu werden, unter anderem deshalb, weil in den Parlamenten vor allem wohlhabende Akademiker*innen sitzen. Doch warum wenden sich viele Bürger*innen, die dieses Gefühl 
haben, gerade Parteien wie der AfD zu, die als Professor*innenpartei begann oder unterstützen superreiche Politiker*innen wie Donald Trump oder Silvio Berlusconi? Die Antwort der Autoren auf diese notorische Frage ist wohl, dass die Politiker*innen der autoritär-populistischen Parteien trotz ihrer persönlichen Hintergründe sich gegen die kosmopolitischen Positionen wenden, die sonst unter gebildeten und wohlhabenden Menschen stärker verbreitet sind. Doch zum Ersten bleibt offen, warum die kommunitaristischere Positionen vertretenden Parteien, die in den letzten Jahren Bedeutung erlangten, gerade einen soziokulturell-konservativen und autoritären Charakter annahmen.

Zum Zweiten stellt sich die Frage, warum ärmere und weniger gebildete Menschen überhaupt so stark kommunitaristischen Positionen zuneigen. Schäfer und Zürn sind der Meinung, dass die demokratischen Defizite eine entscheidende Rolle in den ,,sozialen Mechanismen [spielen, mittels derer] die Globalisierung eine neue Konfliktlinie und mithin den Aufstieg des autoritären Populismus hervorgerufen hat" (S. 84). Dies legt nahe, dass die demokratischen Defizite zumindest teilweise ursächlich für die differierenden politischen Einstellungen sind, um die es in dem Konflikt geht. Es ist auch durchaus plausibel, dass z. B. die Enttäuschung darüber, dass politische Institutionen jenseits der Nationalstaaten häufig gravierende Demokratiedefizite aufweisen, zur Ablehnung internationaler Kooperation und einer stärkeren Betonung der nationalen politischen Gemeinschaften - also zu stärker kommunitaristischen Einstellungen - führen kann. Fehlende demokratische Partizipation und Kontrolle in internationalen Institutionen betreffen jedoch erst einmal auch gebildete und wohlhabende Bürger*innen. Woher kommt also die zunehmende Polarisierung zwischen kommunitaristisch und kosmopolitisch eingestellten Bürger*innen entlang der neuen Konfliktlinie? Wie beschrieben sind nach Schäfer und Zürn die mangelnde Responsivität der Parlamente und die Kompetenzverschiebungen zu nichtmajoritären Institutionen auch deshalb problematisch, weil die Entscheidungsträger*innen in diesen Institutionen stärker kosmopolitische Positionen vertreten als viele wenig gebildete und einkommensschwache Bürger*innen. Das könnte plausibilisieren, warum diese Bevölkerungsgruppen über die demokratischen Defizite stärker enttäuscht sind als andere. Hier wird jedoch bereits vorausgesetzt, dass es die systematischen Unterschiede in den politischen Einstellungen entlang der neuen Konfliktlinie bereits gibt. Denkbar wären höchstens Prozesse, in denen sich demokratische Defizite und differierende politische Einstellungen wechselseitig verstärken.

Aufgrund dieser offenen Fragen bleibt am Ende der Eindruck, dass die politischen Entwicklungen, die Schäfer und Zürn in ihrem sehr lesenswerten Buch beschreiben, zwar zu einer umfassenden Erklärung für den Aufstieg populistischer Parteien beitragen, diese alleine jedoch nicht leisten können. 